\title{
Research Article \\ Some Effects of Side Walls on Unsteady Flow of a Viscous Fluid over a Plane Wall
}

\author{
M. Emin Erdoğan and C. Erdem İmrak \\ İstanbul Teknik Üniversitesi, Makina Fakültesi, 34439 Gümüşsuyu, İstanbul, Turkey \\ Correspondence should be addressed to M. Emin Erdoğan, mkimrak@itu.edu.tr
}

Received 7 May 2009; Revised 9 July 2009; Accepted 7 August 2009

Recommended by Mehrdad Massoudi

Four types of unsteady flows of a viscous fluid over a plane wall bounded by two side walls are considered. They are flow caused by impulsive motion of a plate, flow due to oscillation of a plate, flow induced by constantly accelerating plate, and flow imposed by a plate that applies a constant tangential stress to the fluid. In order to solve these problems, the sine and cosine transformations are used, and exact solutions for the velocity distribution are found in terms of definite integrals. The cases for which the time goes to infinity and the distance between two side walls goes to infinity are compared with the cases for flows over a plane wall in the absence of the side walls. These provide to know the required time to attain the steady-state and what is the distance between the side walls for which the measured value of the velocity or the stress would be unaffected by the presence of the side walls.

Copyright ( 2009 M. E. Erdoğan and C. E. İmrak. This is an open access article distributed under the Creative Commons Attribution License, which permits unrestricted use, distribution, and reproduction in any medium, provided the original work is properly cited.

\section{Introduction}

The flow of a viscous fluid over a plane wall with different boundary and initial conditions has been investigated by many authors. The fluid over a plane wall is initially at rest and is set in motion under the application of a body force considered by Erdoğan [1]. He has shown that there are similarities between the flows considered and Stokes' first and second problems. The flow over a plate, which is initially at rest and is suddenly moved in its own plane with different boundary and initial conditions, has been considered in [2-5]. The flows over a plane wall can be realized due to the impulsive motion of a plate, generated by the constant acceleration of a plate, caused by the oscillation of a plate and due to the application of the tangential stress to the fluid. The extension of these flow systems to the flows in a rotating system [6-9], to the flows of non-Newtonian fluid [10-18] was made by many authors. In [1-18], the authors assumed that the plate extends to infinity. It is well known that the plate is bounded by two side walls and the effect of the side walls is very important. If one wishes to know what is the distance between the side walls for which the measured value of the 
velocity or stress or temperature would be unaffected by the presence of the side walls, it is necessary to investigate the flows over the plate between two side walls. The effects of the side walls for some flows have been considered in [19-25].

The aim of this paper is to investigate the effects of the side walls on the unsteady flow over a plane wall. Four illustrative examples are considered. They are flow due to the impulsive motion of a plate, flow caused by oscillation of a plate, flow due to the constant acceleration of the plate and the flow generated by tangential stress on the fluid. It is well known that the solution of the governing equation for the flow of second-grade fluid over a plane wall obtained by the Laplace transform method is failed [16, 26, 27]. For the flow over a plane wall bounded by two side walls, the shear stress at the bottom wall cannot be calculated by the expression obtained by the Laplace transform method [28-31]. For this reason, in this paper, the Fourier transform method is used.

The first problem considered is the flow due to impulsive motion of a plate. The velocity and the flux across a plane normal to the flow are given in terms of definite integrals. In the limiting cases when time goes to infinity, the solution reduces to the steady-state, and when the distance between two side walls goes to infinity, the solution reduces to the flow over a plate. The second problem considered is the flow due to the oscillation of a plane wall. Two solutions are given in terms of definite integrals. It is important fact that the starting solution can be represented as the sum of the steady-state and transient solutions. For large values of time, the transient solutions disappear. The limiting cases when time goes to infinity, the distance between two side walls goes to infinity and the frequency of the oscillation goes to zero are discussed. The third problem is the flow induced by a constantly accelerating plate. In the limiting case when the distance between the side walls goes to infinity, the solution reduces to the flow over a plate, which can be expressed in terms of a tabulated function. The fourth problem is the flow induced by a plate that applies a constant stress to the fluid. The limiting cases when the time goes to infinity and when the distance between the side walls goes to infinity are discussed. It is a very important fact that these four examples show that the required time to attain the steady-state is affected by the side walls.

\section{Flow due to the Impulsive Motion of a Plane Wall}

The fluid is over a plane wall and between two side walls perpendicular to the plate, The $x$-axis is taken along the plate, the $y$-axis and $z$-axis are perpendicular to the $x$-axis. Flow geometry and coordinate system are illustrated in Figure 1 . The distance between the side walls is $2 b$ and $z$-axis extends to infinity. The governing equation is

$$
\frac{\partial u}{\partial t}=v\left(\frac{\partial^{2} u}{\partial y^{2}}+\frac{\partial^{2} u}{\partial z^{2}}\right)
$$

where $u(y, z, t)$ is the velocity; $v$ is kinematic viscosity of the fluid and $t$ is time. The boundary and initial conditions are

$$
\begin{gathered}
u( \pm b, z, t)=0 \quad \forall t \\
u(y, 0, t)=U \text { for } t>0,-b<y<b \\
u(y, \infty, t)=0 \quad \forall t,-b \leq y \leq b \\
u(y, z, 0)=0 \text { for }-b \leq y \leq b, z>0 .
\end{gathered}
$$



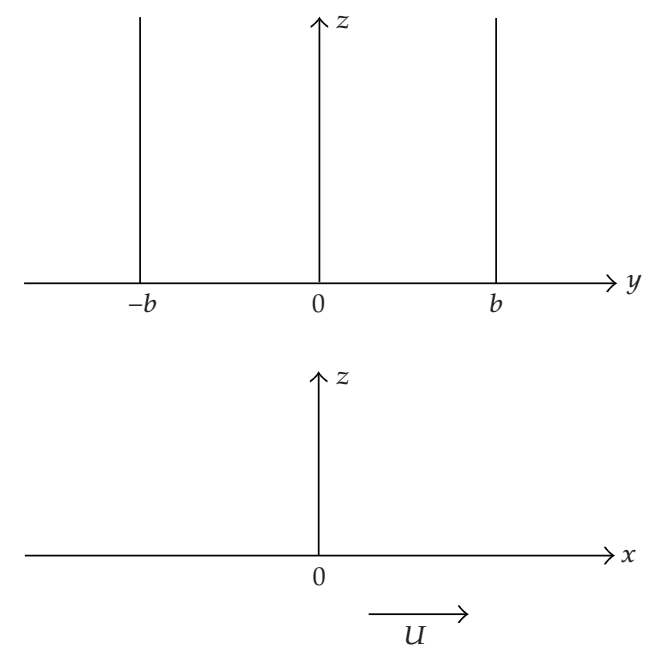

Figure 1: Flow geometry and coordinate system.

The shear stress at the bottom wall cannot be calculated by the expression for velocity obtained by the Laplace transform method [28-31]. For this reason, an expression for velocity by using the sine transform method is obtained.

The first boundary condition suggests that $u(y, z, t)$ can be written in the following form:

$$
\frac{u}{U}=\sum_{n=0} f_{n}(z, t) \cos k_{n} y,
$$

where $k_{n}=(2 n+1) \pi / 2 b$. The sine transform of $f_{n}(z, t)[32]$ is

$$
\bar{f}_{n}=\int_{0}^{\infty} f_{n} \sin \lambda z d z
$$

and $\bar{f}_{n}(0)=0$. The problem reduces to the solution of the following ordinary differential equation:

$$
\bar{f}_{n}^{\prime}+v\left(k_{n}^{2}+\lambda^{2}\right) \bar{f}_{n}=\frac{4(-1)^{n} v \lambda}{(2 n+1) \pi}
$$

where a prime denotes differentiation with respect to $t$. The solution is

$$
\bar{f}_{n}=\frac{4(-1)^{n}}{(2 n+1) \pi} \frac{\lambda}{\lambda^{2}+k_{n}^{2}}\left[1-e^{-\left(k_{n}^{2}+\lambda^{2}\right) v t}\right]
$$


The inverse of $\bar{f}_{n}$ is given by the relation [32]

$$
f_{n}=\frac{2}{\pi} \int_{0}^{\infty} \bar{f}_{n} \sin \lambda z d \lambda
$$

and inserting the expression for $\bar{f}_{n}$ into this equation, one finds

$$
f_{n}=\frac{8(-1)^{n}}{(2 n+1) \pi^{2}} \int_{0}^{\infty} \frac{\lambda \sin \lambda z}{\lambda^{2}+k_{n}^{2}} d \lambda-\frac{8(-1)^{n}}{(2 n+1) \pi^{2}} \int_{0}^{\infty} \frac{\lambda \sin \lambda z}{\lambda^{2}+k_{n}^{2}} e^{-\left(k_{n}^{2}+\lambda^{2}\right) v t} d \lambda
$$

The first integral is

$$
\int_{0}^{\infty} \frac{\lambda \sin \lambda z}{\lambda^{2}+k_{n}^{2}} d \lambda=\frac{\pi}{2} e^{-k_{n} z}
$$

and since [28]

$$
\frac{4}{\pi} \sum_{n=0} \frac{(-1)^{n}}{2 n+1} e^{-k_{n} z} \cos k_{n} y=1-\frac{2}{\pi} \int_{0}^{\infty} \frac{\cosh \lambda y}{\lambda \cosh \lambda b} \sin \lambda z d \lambda
$$

the velocity becomes

$$
\frac{u}{U}=1-\frac{2}{\pi} \int_{0}^{\infty} \frac{\cosh \lambda y}{\lambda \cosh \lambda b} \sin \lambda z d \lambda-\frac{4}{\pi} \sum_{n=0} \frac{(-1)^{n} \cos k_{n} y}{k_{n} b} \int_{0}^{\infty} \frac{\lambda \sin \lambda z}{\lambda^{2}+k_{n}^{2}} e^{-\left(k_{n}^{2}+\lambda^{2}\right) v t} d \lambda,
$$

or

$$
\begin{aligned}
\frac{u}{U}= & \frac{2}{\pi} \tan ^{-1} \frac{\cos (\pi y / 2 b)}{\sinh (\pi z / 2 b)}-\frac{2}{\pi} \sum_{n=0} \frac{(-1)^{n} \cos k_{n} y}{2 n+1} \\
& \times\left\{2 e^{-k_{n} z}-\left[e^{-k_{n} z} \operatorname{erfc}\left(\frac{z}{2 \sqrt{v t}}-k_{n} \sqrt{v t}\right)+e^{k_{n} z} \operatorname{erfc}\left(\frac{z}{2 \sqrt{v t}}+k_{n} \sqrt{v t}\right)\right]\right\} .
\end{aligned}
$$

In the limiting case, when $t$ goes to infinity, (2.11) reduces to

$$
\frac{u}{U}=1-\frac{2}{\pi} \int_{0}^{\infty} \frac{\cosh \lambda y}{\lambda \cosh \lambda b} \sin \lambda z d \lambda
$$

This is the expression for velocity in the case of the steady-state.

When $b$ goes to infinity in (2.11), since

$$
\int_{0}^{\infty} \frac{\sin \lambda z}{\lambda} e^{-\lambda^{2} v t} d \lambda=\frac{\pi}{2} \operatorname{erf} \frac{z}{2 \sqrt{v t}}
$$




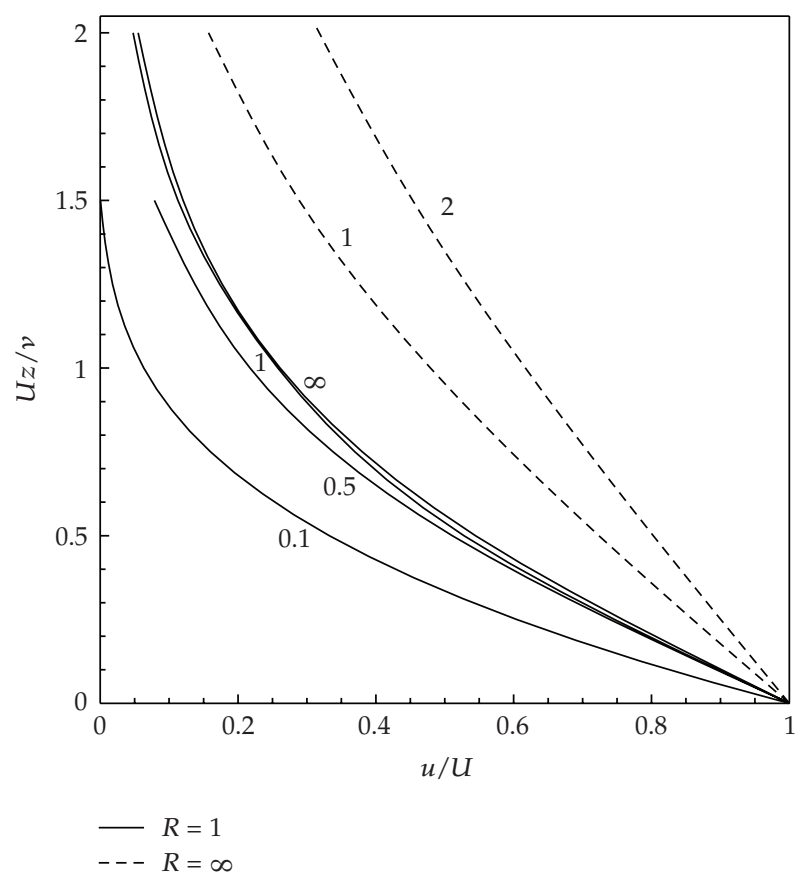

Figure 2: The variation of $u / U$ with $z / b$ for various values of $v t / b^{2}$, at $y=0$, with and without side walls; $R=U b / v$.

one finds

$$
\frac{u}{U}=\operatorname{erfc} \frac{z}{2 \sqrt{v t}}
$$

The variation of $u / U$ with $z / b$ for various values of $v t / b^{2}$, at $y=0$, with and without side walls is illustrated in Figure 2. It is clearly seen from the figure that the required time to attain the steady-state in the presence of the side walls is shorter than that of the case in the absence of the side walls.

The volume flux across a plane normal to the flow is given by

$$
Q=\int_{-b}^{b} \int_{0}^{\infty} u d y d z
$$

Inserting (2.11) into this equation and using the integral [33]

$$
\int_{0}^{\infty} \sin \lambda z d \lambda=\frac{1}{z}
$$

and the integral

$$
\int_{0}^{\infty} \frac{e^{-\left(\lambda^{2}+k_{n}^{2}\right) v t}}{\lambda^{2}+k_{n}^{2}} d \lambda=\frac{\pi}{2 k_{n}} \operatorname{erfc}\left(k_{n} \sqrt{v t}\right),
$$


one finds

$$
\frac{Q}{U b^{2}}=\frac{4}{\pi} F(\infty)-4 \sum_{n=0} \frac{1}{b^{3} k_{n}^{3}} \operatorname{erfc}\left(k_{n} \sqrt{v t}\right)
$$

where $F(\infty)$ is given by a definite integral

$$
F(\infty)=\int_{0}^{\infty} \frac{\xi-\tanh \xi}{\xi^{3}} d \xi
$$

and the numerical value of $F(\infty)$ is 0.8525867976 correct to ten decimal places [30]. The comparison of the values of the volume flux in the case of the steady-state gives

$$
F(\infty)=\frac{8}{\pi^{2}} \sum_{n=0}(2 n+1)^{-3}
$$

The shear stress at the bottom wall can be calculated by (2.11). Taking the derivative of (2.11) with respect to zand then putting $z=0$, one finds

$$
\frac{\left(\sigma_{x z}\right)_{z=0}}{\rho U^{2}}=-\left[\frac{v}{U b \cos \pi(y /(2 b))}+2 \frac{v}{U} \sum_{n=0} \frac{(-1)^{n} \cos k_{n} y}{b k_{n} \sqrt{v t}} \operatorname{ierfc}\left(k_{n} \sqrt{v t}\right)\right],
$$

where

$$
\operatorname{ierfc} x=\int_{x}^{\infty} \operatorname{erfc} \xi d \xi
$$

is the integral of the complementary error function, which can be obtained from the tables [34]. When $t$ goes to infinity since ierfc $(\infty)=0,(2.22)$ reduces to the expression for the steady-state

$$
\frac{\left(\sigma_{x z}\right)_{z=0}}{\rho U^{2}}=-\frac{v}{U b \cos \pi(y /(2 b))} .
$$

In the absence of the side walls, namely, when $b$ goes to infinity, since ierfc $(0)=1 / \sqrt{\pi},(2.22)$ reduces to

$$
\frac{\left(\sigma_{x z}\right)_{z=0}}{\rho U^{2}}=-\frac{1}{U} \sqrt{\frac{\nu}{\pi t}}
$$




\section{Flow due to Oscillation of a Plane Wall}

The flow over a plane wall which is initially at rest and the plate begins to oscillate in its own plane is termed Stokes' second problem [35]. The $x$-axis is taken along the plate; the $y$-axis and $z$-axis are perpendicular to the $x$-axis. The distance between the side walls is $2 b$, and the $z$-axis extends to infinity. The governing equation is (2.1). The boundary and initial conditions are

$$
\begin{gathered}
u( \pm b, z, t)=0 \quad \forall t \\
u(y, 0, t)=U \cos \omega t \text { or } U \sin \omega t \text { for } t>0,-b<y<b, \\
u(y, \infty, t)=0 \quad \forall t,-b \leq y \leq b, \\
u(y, z, 0)=0 \text { for }-b \leq y \leq b,-z>0 .
\end{gathered}
$$

The first boundary condition suggests that $u(y, z, t)$ can be written in the following form:

$$
\frac{u}{U}=\sum_{n=0} f_{n}(z, t) \cos k_{n} y
$$

where $k_{n}=(2 n+1) \pi / 2 b$. The sine transform of $f_{n}(z, t)$ is

$$
\bar{f}_{n}=\int_{0}^{\infty} f_{n} \sin \lambda z d z
$$

and $\bar{f}_{n}(0)=0$. The problem reduces to the solution of the following ordinary differential equation:

$$
\bar{f}_{n}^{\prime}+v\left(k_{n}^{2}+\lambda^{2}\right) \bar{f}_{n}=\frac{4(-1)^{n} v \lambda}{(2 n+1) \pi} \cos \omega t
$$

where a prime denotes differentiation with respect to $t$. The solution is

$$
\begin{gathered}
\bar{f}_{n}=\frac{4(-1)^{n}}{(2 n+1) \pi}\left\{\frac{v^{2} \lambda\left(\lambda^{2}+k_{n}^{2}\right)}{\omega^{2}+v^{2}\left(\lambda^{2}+k_{n}^{2}\right)^{2}} \cos \omega t+\frac{\omega v \lambda}{\omega^{2}+v^{2}\left(\lambda^{2}+k_{n}^{2}\right)^{2}} \sin \omega t\right. \\
\left.-\frac{v^{2} \lambda\left(\lambda^{2}+k_{n}^{2}\right)}{\omega^{2}+v^{2}\left(\lambda^{2}+k_{n}^{2}\right)^{2}} e^{-\left(\lambda^{2}+k_{n}^{2}\right) v t}\right\}
\end{gathered}
$$

respectively,

$$
\begin{aligned}
\bar{f}_{n}=\frac{4(-1)^{n}}{(2 n+1) \pi}\{ & -\frac{\omega v \lambda}{\omega^{2}+\nu^{2}\left(\lambda^{2}+k_{n}^{2}\right)^{2}} \cos \omega t+\frac{v^{2} \lambda\left(\lambda^{2}+k_{n}^{2}\right)}{\omega^{2}+v^{2}\left(\lambda^{2}+k_{n}^{2}\right)^{2}} \sin \omega t \\
& \left.+\frac{\omega v \lambda}{\omega^{2}+v^{2}\left(\lambda^{2}+k_{n}^{2}\right)^{2}} e^{-\left(\lambda^{2}+k_{n}^{2}\right) v t}\right\},
\end{aligned}
$$


The inverse of $\bar{f}_{n}$ is given by the relation

$$
f_{n}=\frac{2}{\pi} \int_{0}^{\infty} \bar{f}_{n} \sin \lambda z d \lambda
$$

and inserting the expression for $\bar{f}_{n}$ into this equation, one finds

$$
\begin{aligned}
f_{n}=\frac{8(-1)^{n}}{(2 n+1) \pi^{2}}[ & (\cos \omega t) \int_{0}^{\infty} \frac{v^{2} \lambda\left(k_{n}^{2}+\lambda^{2}\right)}{\omega^{2}+\nu^{2}\left(k_{n}^{2}+\lambda^{2}\right)^{2}} \sin \lambda z d \lambda \\
& +(\sin \omega t) \int_{0}^{\infty} \frac{\omega v \lambda}{\omega^{2}+v^{2}\left(k_{n}^{2}+\lambda^{2}\right)^{2}} \sin \lambda z d \lambda \\
& \left.\quad-\int_{0}^{\infty} \frac{v^{2} \lambda\left(k_{n}^{2}+\lambda^{2}\right)}{\omega^{2}+v^{2}\left(k_{n}^{2}+\lambda^{2}\right)^{2}} e^{-\left(k_{n}^{2}+\lambda^{2}\right) v t} \sin \lambda z d \lambda\right]
\end{aligned}
$$

respectively,

$$
\begin{aligned}
f_{n}=\frac{8(-1)^{n}}{(2 n+1) \pi^{2}}[- & (\cos \omega) t \int_{0}^{\infty} \frac{\omega v \lambda}{\omega^{2}+v^{2}\left(k_{n}^{2}+\lambda^{2}\right)^{2}} \sin \lambda z d \lambda \\
& +(\sin \omega t) \int_{0}^{\infty} \frac{v^{2} \lambda\left(k_{n}^{2}+\lambda^{2}\right)}{\omega^{2}+\nu^{2}\left(k_{n}^{2}+\lambda^{2}\right)^{2}} \sin \lambda z d \lambda \\
& \left.+\int_{0}^{\infty} \frac{\omega v \lambda}{\omega^{2}+v^{2}\left(k_{n}^{2}+\lambda^{2}\right)^{2}} e^{-\left(k_{n}^{2}+\lambda^{2}\right)^{2} v t} \sin \lambda z d \lambda\right] .
\end{aligned}
$$

Since [36]

$$
\begin{aligned}
& \int_{0}^{\infty} \frac{\lambda \sin \lambda z}{\omega^{2}+v^{2}\left(k_{n}^{2}+\lambda^{2}\right)^{2}} d \lambda=\frac{\pi}{2 \omega v} e^{-A z} \sin B z, \\
& \int_{0}^{\infty} \frac{\lambda\left(k_{n}^{2}+\lambda^{2}\right) \sin \lambda z}{\omega^{2}+v^{2}\left(k_{n}^{2}+\lambda^{2}\right)^{2}} d \lambda=\frac{\pi}{2 v^{2}} e^{-A z} \cos B z,
\end{aligned}
$$

where

$$
2 A^{2}=\sqrt{k_{n}^{4}+\frac{\omega^{2}}{v^{2}}}+k_{n}^{2}, \quad 2 B^{2}=\sqrt{k_{n}^{4}+\frac{\omega^{2}}{v^{2}}}-k_{n}^{2}
$$

inserting the integrals into (3.8) and (3.9), one finds

$$
f_{n}=\frac{4(-1)^{n}}{(2 n+1) \pi}\left[e^{-A z} \cos (\omega t-B z)-\frac{2}{\pi} \int_{0}^{\infty} \frac{v^{2} \lambda\left(k_{n}^{2}+\lambda^{2}\right) \sin \lambda z}{\omega^{2}+v^{2}\left(k_{n}^{2}+\lambda^{2}\right)^{2}} e^{-\left(k_{n}^{2}+\lambda^{2}\right) v t} d \lambda\right],
$$


respectively,

$$
f_{n}=\frac{4(-1)^{n}}{(2 n+1) \pi}\left[e^{-A z} \sin (\omega t-B z)+\frac{2}{\pi} \int_{0}^{\infty} \frac{\omega v \lambda \sin \lambda z}{\omega^{2}+v^{2}\left(k_{n}^{2}+\lambda^{2}\right)^{2}} e^{-\left(k_{n}^{2}+\lambda^{2}\right) v t} d \lambda\right] .
$$

In the limiting case, where $t$ goes to infinity, (3.8) and (3.9) reduce to

$$
f_{n}=\frac{4(-1)^{n}}{(2 n+1) \pi}\left[e^{-A z} \cos (\omega t-B z)\right]
$$

respectively,

$$
f_{n}=\frac{4(-1)^{n}}{(2 n+1) \pi}\left[e^{-A z} \sin (\omega t-B z)\right]
$$

When $b$ goes to infinity, (3.8) and (3.9) reduce to [5]

$$
\frac{u}{U}=e^{-\sqrt{(\omega / 2 v)} z} \cos \left(\omega t-\sqrt{\frac{\omega}{2 v}} z\right)
$$

respectively,

$$
\frac{u}{U}=e^{-\sqrt{(\omega / 2 v) z}} \sin \left(\omega t-\sqrt{\frac{\omega}{2 v} z}\right)
$$

The variation of $u / U$ with $(\omega / v)^{1 / 2} z$ for various values of $\omega t$ at $y=0$, with and without side walls is illustrated in Figure 3. It is obvious that the required time to attain the steady-state for the cosine oscillation of the plate is shorter than for the sine oscillation of it. There is an appreciable effect of the side walls on the disappearance of the transients. More details were given in $[2,31]$. The first terms in (3.12) and (3.13) show the steady-state and the second terms denote transients. When $t$ goes to infinity the transients disappear. The integrals in (3.12) and (3.13) can be written as

$$
\begin{aligned}
& \int_{0}^{\infty} \frac{\lambda\left(k_{n}^{2}+\lambda^{2}\right) \sin \lambda z}{\omega^{2}+v^{2}\left(k_{n}^{2}+\lambda^{2}\right)^{2}} e^{-\left(k_{n}^{2}+\lambda^{2}\right) v t} d \lambda=\frac{e^{-k_{n}^{2} v t}}{2 v^{2}}(F+G), \\
& \int_{0}^{\infty} \frac{\lambda \sin \lambda z}{\omega^{2}+v^{2}\left(k_{n}^{2}+\lambda^{2}\right)^{2}} e^{-\left(k_{n}^{2}+\lambda^{2}\right) v t} d \lambda=\frac{i e^{-k_{n}^{2} v t}}{2 \omega v}(F-G),
\end{aligned}
$$




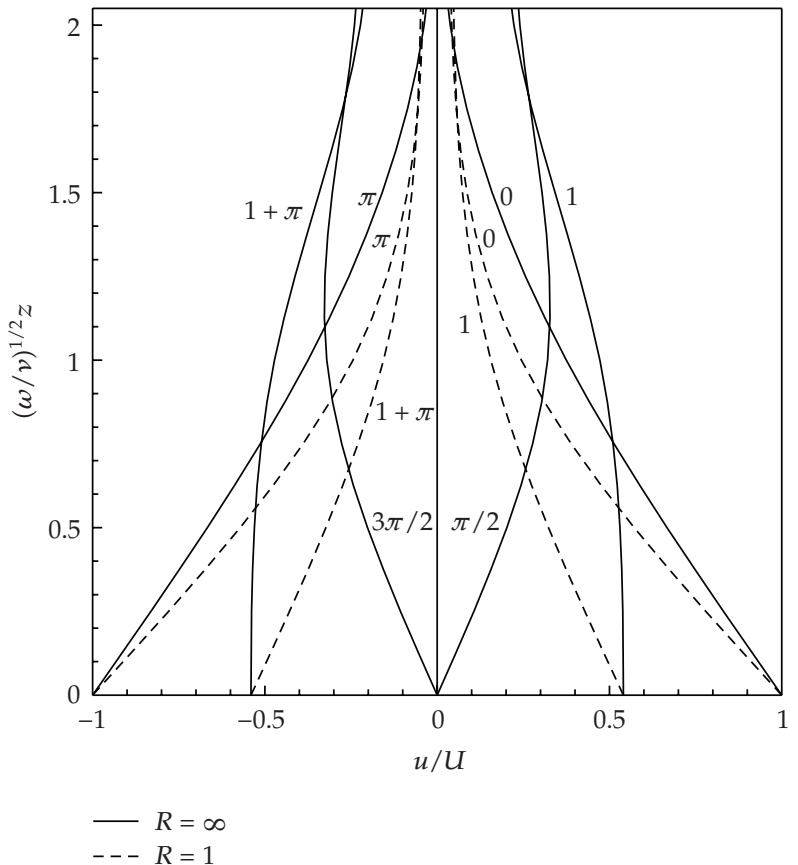

Figure 3: The variation of $u / U$ with $(\omega / v)^{1 / 2} z$ for various values of $\omega t$, at $y=0$, with and without side walls. $R=\omega b^{2} / v$.

where

$$
\begin{gathered}
F=\int_{0}^{\infty} \frac{\lambda \sin \lambda z}{\lambda^{2}+\beta^{2}} e^{-\lambda^{2} v t} d \lambda, \quad G=\int_{0}^{\infty} \frac{\lambda \sin \lambda z}{\lambda^{2}+\alpha^{2}} e^{-\lambda^{2} v t} d \lambda \\
\alpha^{2}=k_{n}^{2}-i \frac{\omega}{v}, \quad \beta^{2}=k_{n}^{2}+i \frac{\omega}{v}, \\
\int_{0}^{\infty} \frac{x \sin b x}{c^{2}+x^{2}} e^{-a x^{2}} d x=\frac{\pi}{4} e^{a c^{2}}\left[2 e^{-b c}-e^{b c} \operatorname{erfc}\left(\frac{b}{2 \sqrt{a}}+c \sqrt{a}\right)-e^{-b c} \operatorname{erfc}\left(\frac{b}{2 \sqrt{a}}-c \sqrt{a}\right)\right] .
\end{gathered}
$$

\section{Flow Induced by a Constantly Accelerating Plate}

The plate is initially at rest and has, after time zero, a constant acceleration $A$. The governing equation is given by (2.1). The boundary and initial conditions are

$$
\begin{gathered}
u( \pm b, z, t)=0 \quad \forall t, \\
u(y, 0, t)=A t \quad \text { for } t>0,-b<y<b, \\
u(y, \infty, t)=0 \quad \forall t,-b \leq y \leq b, \\
u(y, z, 0)=0 \text { for }-b \leq y \leq b, z>0 .
\end{gathered}
$$


The first boundary condition suggests that $u(y, z, t)$ can be written in the following form:

$$
u=A \sum_{n=0} f_{n}(z, t) \cos k_{n} y,
$$

where $k_{n}=(2 n+1) \pi / 2 b$. The sine transform of $f_{n}(z, t)$ is $\bar{f}_{n}(t)$ and the initial condition becomes $\bar{f}_{n}(0)=0$. The problem reduces to the solution of the following ordinary differential equation:

$$
\bar{f}_{n}^{\prime}+v\left(k_{n}^{2}+\lambda^{2}\right) \bar{f}_{n}=\frac{4(-1)^{n}}{(2 n+1) \pi} \lambda v t
$$

where a prime denotes differentiation with respect to $t$. The solution is

$$
\bar{f}_{n}=\frac{4(-1)^{n}}{(2 n+1) \pi}\left\{\frac{\lambda t}{k_{n}^{2}+\lambda^{2}}-\frac{\lambda}{v\left(k_{n}^{2}+\lambda^{2}\right)^{2}}\left[1-e^{-\left(k_{n}^{2}+\lambda^{2}\right) v t}\right]\right\} .
$$

The inverse of $\bar{f}_{n}$ is given by the relation

$$
f_{n}=\frac{2}{\pi} \int_{0}^{\infty} \bar{f}_{n} \sin \lambda z d \lambda
$$

and inserting the expression for $\bar{f}_{n}$ into this equation, one finds

$$
f_{n}=\frac{8(-1)^{n}}{(2 n+1) \pi^{2}}\left[\frac{\pi}{2} t-\left(k_{n}^{2} t\right) \int_{0}^{\infty} \frac{\sin \lambda z}{\lambda\left(k_{n}^{2}+\lambda^{2}\right)} d \lambda-\frac{1}{v} \int_{0}^{\infty} \frac{\lambda \sin \lambda z}{\left(k_{n}^{2}+\lambda^{2}\right)^{2}}\left[1-e^{-\left(k_{n}^{2}+\lambda^{2}\right) v t}\right] d \lambda\right] .
$$

In the limiting case when $b$ goes to infinity, one obtains

$$
\lim _{b \rightarrow \infty} \frac{u}{A}=t-\frac{2}{\pi} \int_{0}^{\infty} \frac{\sin \lambda z}{v \lambda^{3}}\left(1-e^{-\lambda^{2} v t}\right) d \lambda
$$

or in nondimensional form

$$
\frac{u}{(A v)^{1 / 3}}=4 \tau i^{2} \operatorname{erfc} \frac{\zeta}{2 \sqrt{\tau}}
$$

where $\tau=\left(A^{2} / v\right)^{1 / 3} t, \zeta=\left(A / v^{2}\right)^{1 / 3} z$ and $(A v)^{1 / 3}$ is the characteristic velocity, $\left(v^{2} / A\right)^{1 / 3}$ is the characteristic length and $\left(v / A^{2}\right)^{1 / 3}$ is the characteristic time. $i^{2} \operatorname{erfc} x$ is the integral of the complementary error function, which can be obtained from the tables [34]. The variation of $u /(A v)^{1 / 3}$ with $\left(A / v^{2}\right)^{1 / 3} z$ for various values of $\left(v / A^{2}\right)^{1 / 3} t$, at $y=0$ is illustrated in Figure 4. It is clearly seen from the figure that the required time to attain to the flow without the side walls is short. 


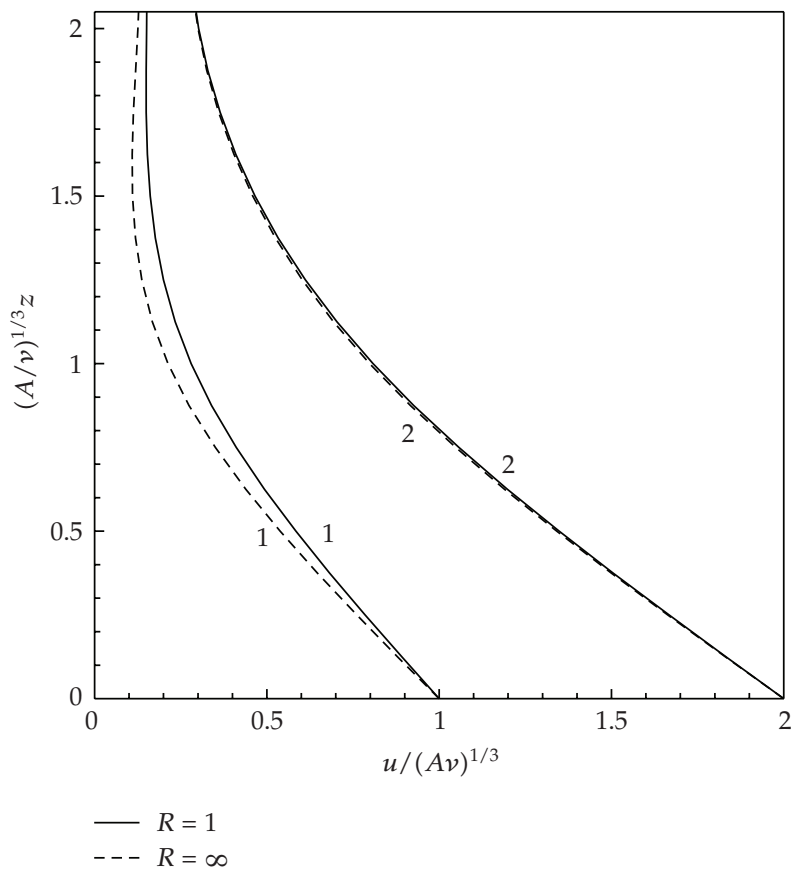

Figure 4: The variation of $u /(A v)^{1 / 3}$ with $\left(A / v^{2}\right)^{1 / 3} z$ for various values of $\left(v / A^{2}\right)^{1 / 3} t$, at $y=0$, with and without side walls. $R=b /\left(v^{2} / A\right)^{1 / 3}$.

\section{Flow Induced by a Plate that Applies a Constant Stress}

Suppose that the plane wall suddenly applies a constant tangential stress $\sigma$ to the fluid. The governing equation is (2.1), and the boundary and initial conditions are

$$
\begin{gathered}
u( \pm b, z, t)=0 \quad \forall t, \\
u(y, \infty, t)=0 \quad \forall t,-b \leq y \leq b, \\
u(y, z, 0)=0 \quad \text { for }-b \leq y \leq b, z>0, \\
\mu\left(\frac{\partial u}{\partial z}\right)_{z=0}=-\sigma .
\end{gathered}
$$

The minus sign appears, and $\sigma$ represents the tangential stress exerted on the fluid by the plate. The first boundary condition suggests that $u(y, z, t)$ can be written in the following form:

$$
u=\sum_{n=0} f_{n}(z, t) \cos k_{n} y
$$


where $k_{n}=(2 n+1) \pi / 2 b$. The cosine transform of $f_{n}(z, t)$ is

$$
\bar{f}_{n}=\int_{0}^{\infty} f_{n} \cos \lambda z d z
$$

and the initial condition is $\bar{f}_{n}(0)=0$. The problem reduces to the solution of the following ordinary differential equation:

$$
\bar{f}_{n}^{\prime}+v\left(k_{n}^{2}+\lambda^{2}\right) \bar{f}_{n}=\frac{4(-1)^{n}}{(2 n+1) \pi} \frac{\sigma}{\rho}
$$

where a prime denotes differentiation with respect to $t$. The solution is

$$
\bar{f}_{n}=\frac{4(-1)^{n}}{(2 n+1) \pi} \frac{\sigma}{\rho v} \frac{1}{k_{n}^{2}+\lambda^{2}}\left[1-e^{-\left(k_{n}^{2}+\lambda^{2}\right) v t}\right]
$$

The inverse of $\bar{f}_{n}$ is given by the relation [32]

$$
f_{n}=\frac{2}{\pi} \int_{0}^{\infty} \bar{f}_{n} \cos \lambda z d \lambda
$$

and inserting the expression for $\bar{f}_{n}$ into this equation, one finds

$$
f_{n}=\frac{8(-1)^{n} \sigma}{(2 n+1) \pi^{2} \rho v} \int_{0}^{\infty} \frac{\cos \lambda z}{k_{n}^{2}+\lambda^{2}}\left[1-e^{-\left(k_{n}^{2}+\lambda^{2}\right) v t}\right] d \lambda .
$$

The velocity $u$ becomes

$$
u=\frac{8 \sigma}{\rho v \pi^{2}} \sum_{n=0} \frac{(-1)^{n} \cos k_{n} y}{2 n+1} \int_{0}^{\infty} \frac{\cos \lambda z}{k_{n}^{2}+\lambda^{2}}\left[1-e^{-\left(k_{n}^{2}+\lambda^{2}\right) v t}\right] d \lambda
$$

or

$$
u=\frac{8 \sigma}{\rho v \pi^{2}} \sum_{n=0} \frac{(-1)^{n} \cos k_{n} y}{2 n+1}\left[\frac{\pi}{2 k_{n}}\left(e^{-k_{n} z}-1\right)+\int_{0}^{\infty} \frac{1-e^{-\left(k_{n}^{2}+\lambda^{2}\right) v t} \cos \lambda z}{k_{n}^{2}+\lambda^{2}} d \lambda\right]
$$

The variation of $u /(\sigma / \rho)^{1 / 2}$ with $z /\left(\rho v^{2} / \sigma\right)^{1 / 2}$ for various values of $(\sigma / \rho v) t$, at $y=0$, is illustrated in Figure 5. It is clearly seen from the figure that for large times, the flow becomes steady in the case of the side walls, but the flow remains time-dependent in the absence of the side walls. When $b$ goes to infinity, (5.9) becomes

$$
u=\frac{\sigma}{\rho v}\left(-z+\frac{2}{\pi} \int_{0}^{\infty} \frac{1-e^{-\lambda^{2} v t} \cos \lambda z}{\lambda^{2}} d \lambda\right) .
$$




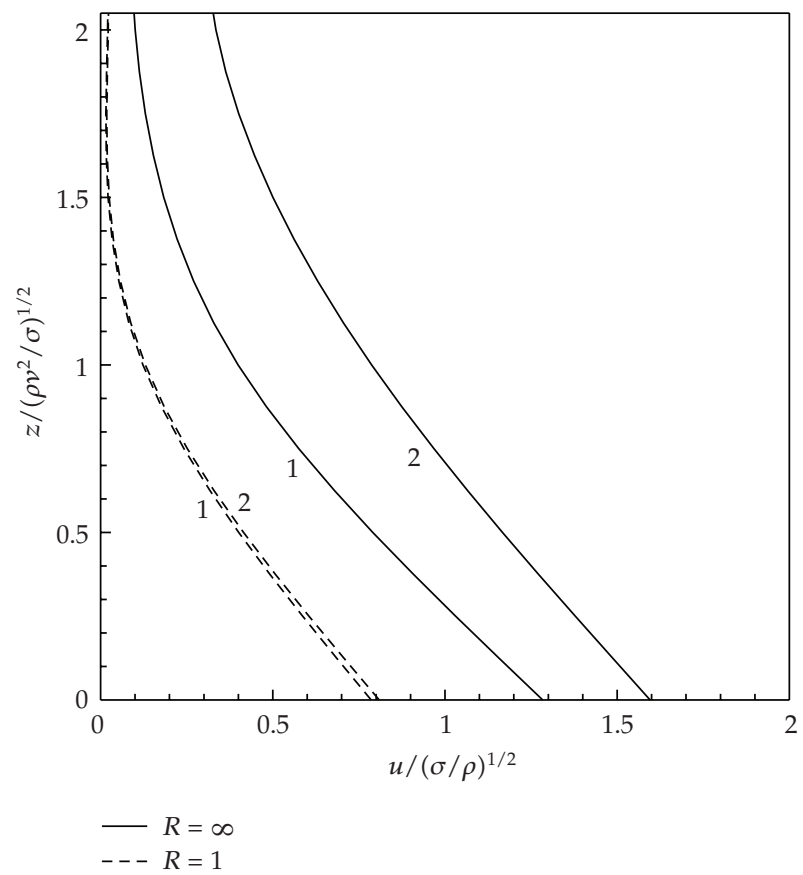

Figure 5: The variation of $u /(\sigma / \rho)^{1 / 2}$ with $z /\left(\rho v^{2} / \sigma\right)^{1 / 2}$ for various values of $(\sigma / \rho v) t$, at $y=0$, with and without side walls. $R=b /\left(\rho v^{2} / \sigma\right)^{1 / 2}$.

By using the equality

$$
\int_{0}^{\infty} \frac{1-e^{-\lambda^{2} v t} \cos \lambda z}{\lambda^{2}} d \lambda=\frac{\pi}{2} z+\sqrt{\pi v t} \operatorname{ierfc} \frac{z}{2 \sqrt{v t}}
$$

one finds

$$
u=2 \frac{\sigma}{\rho v} \sqrt{v t} \operatorname{ierfc} \frac{z}{2 \sqrt{v t}}
$$

where ierfc $x$ is he integral of the complementary error function, which can be obtained from the tables [34].

\section{Conclusions}

Four types of unsteady flows of incompressible viscous fluids over a plane wall bounded by two side walls are considered. In order to understand the effects of the side walls, the cases for which time goes to infinity and the distance between two side walls goes to infinity are compared with the cases for flows over a plane wall in the absence of the side walls. These provide to know the required time to attain the steady-state and what is the distance between the side walls for which the measured value of the velocity or the stress or the temperature would be unaffected by the presence of the side walls. The flow problems which are the flow 
generated by impulsive motion of a plate, flow due to oscillation of the plate, flow induced by constantly accelerating plate, and flow due to imposed by a plate that applies a constant tangential stress to the fluid are solved by the application of the Fourier transform method to the governing equation of motion. The solutions can be expressed in terms of the definite integrals. It is shown that the starting solution for the flow of the oscillating plate can be presented as the sum of the steady-state and the transient solutions. The limiting cases when the time goes to infinity and the distance between two side walls goes to infinity are obtained and discussed. For flow induced by a plate that applies a constant stress, for large times the flow becomes steady in the case of the side walls, but the flow remains time-dependent in the absence of the side walls.

\section{Acknowledgments}

The authors are grateful to Professor K. R. Rajagopal for many stimulating correspondences and suggestions. The authors would like to thank the referees for their valuable comments.

\section{References}

[1] M. E. Erdoğan, "Unsteady flow of a viscous fluid over a plane wall," Zeitschrift für Angewandte Mathematik und Mechanik, vol. 77, no. 10, pp. 733-740, 1997.

[2] M. E. Erdoğan, "A note on an unsteady flow of a viscous fluid due to an oscillating plane wall," International Journal of Non-Linear Mechanics, vol. 35, no. 1, pp. 1-6, 2000.

[3] Y. Zeng and S. Weinbaum, "Stokes problems for moving half-planes," Journal of Fluid Mechanics, vol. 287, pp. 59-74, 1995.

[4] C. Fetecáu, D. Vieru, and C. Fetecáu, "A note on the second problem of Stokes for Newtonian fluids," International Journal of Non-Linear Mechanics, vol. 43, pp. 451-457, 2008.

[5] M. E. Erdoğan and C. E. İmrak, "On the comparison of the solutions obtained by using two different transform methods for the second problem of Stokes for Newtonian fluids," International Journal of Non-Linear Mechanics, vol. 44, no. 1, pp. 27-30, 2009.

[6] P. Puri and P. K. Kulshrestha, "Unsteady hydromagnetic boundary layer in a rotating medium," Journal of Applied Mechanics, vol. 43, no. 2, pp. 205-208, 1976.

[7] L. Debnath, "On unsteady magnetohydrodynamic boundary layers in a rotating flow, Recent Research on Unsteady Boundary Layers," in Proceedings of the IUTAM Symposium, pp. 1397-1421, 1971.

[8] L. Debnath and M. Hull, "On unsteady Stokes, Ekman and Rayleigh layers in a rotating fluid," Bulletin de l'Académie Polonaise des Sciences, vol. 21, pp. 141-149, 1973.

[9] C. Thornly, "On Stokes and Rayleigh layers in a rotating system," The Quarterly Journal of Mechanics and Applied Mathematics, vol. 21, pp. 451-461, 1968.

[10] T. W. Ting, "Certain non-steady flows of second-order fluids," Archive for Rational Mechanics and Analysis, vol. 14, no. 1, pp. 1-26, 1963.

[11] I. Teipel, "The impulsive motion of a flat plate in a viscoelastic fluid," Acta Mechanica, vol. 39, no. 3-4, pp. 277-279, 1981.

[12] K. R. Rajagopal, “A note on unsteady unidirectional flows of a non-Newtonian fluid," International Journal of Non-Linear Mechanics, vol. 17, no. 5-6, pp. 369-373, 1982.

[13] M. E. Erdoğan, "Plane surface suddenly set in motion in a non-Newtonian fluid," Acta Mechanica, vol. 108, no. 1-4, pp. 179-187, 1995.

[14] R. I. Tanner, "Note on the Rayleigh problem for a visco-elastic fluid," Zeitschrift für Angewandte Mathematik und Physik, vol. 13, no. 6, pp. 573-580, 1962.

[15] M. E. Erdoğan, "On unsteady motions of a second-order fluid over a plane wall," International Journal of Non-Linear Mechanics, vol. 38, no. 7, pp. 1045-1051, 2003.

[16] R. Bandell, K. R. Rajagopal, and C. P. Galdi, "On some unsteady motions of fluids of second grade," Archives of Mechanics, vol. 47, pp. 661-676, 1995. 
[17] C. Fetecáu and J. Zierep, "On a class of exact solutions of the equations of motion of a second grade fluid," Acta Mechanica, vol. 150, no. 1-2, pp. 135-138, 2001.

[18] C. Fetecáu and C. Fetecáu, "The Rayleigh-Stokes problem for heated second grade fluids," International Journal of Non-Linear Mechanics, vol. 37, no. 6, pp. 1011-1015, 2002.

[19] M. E. Erdoğan, "Effects of the side walls in generalized Couette flow," Journal of Applied Mechanics and Engineering, vol. 3, pp. 271-286, 1998.

[20] M. E. Erdoğan, "The effects of side walls on axial flow in rectangular ducts with suction and injection," Acta Mechanica, vol. 162, no. 1-4, pp. 157-166, 2003.

[21] M. E. Erdoğan and C. E. İmrak, "Effect of the side walls on the unsteady flow in A duct of rectangular cross-section," Mathematical and Computational Applications, vol. 9, no. 3, pp. 443-448, 2004.

[22] M. E. Erdoğan and C. E. İmrak, "Effects of the wall curvature on the flow in a duct of uniform crosssection," Acta Mechanica, vol. 167, no. 3-4, pp. 123-130, 2004.

[23] M. E. Erdoğan and C. E. İmrak, "Effects of the side walls on the unsteady flow of a second-grade fluid in a duct of uniform cross-section," International Journal of Non-Linear Mechanics, vol. 39, no. 8, pp. 1379-1384, 2004.

[24] M. E. Erdoğan and C. E. İmrak, "Effects of the side walls on starting flows in ducts," International Journal of Non-Linear Mechanics, vol. 40, no. 1, pp. 103-111, 2005.

[25] M. E. Erdoğan and C. E. İmrak, "The effects of the side walls on the flow of a second grade fluid in ducts with suction and injection," International Journal of Non-Linear Mechanics, vol. 42, no. 5, pp. 765-772, 2007.

[26] V. M. Soundalgekar, "Stokes problem for elastico-viscous fluid," Rheologica Acta, vol. 13, no. 2, pp. 177-179, 1974.

[27] P. Puri, "Impulsive motion of a flat plate in a Rivlin-Ericksen fluid," Rheologica Acta, vol. 23, no. 4, pp. 451-453, 1984.

[28] M. E. Erdoğan, "Effects of the side walls on the flow over a moving plane wall," International Journal of Applied Mechanics and Engineering, vol. 2, pp. 415-432, 1997.

[29] G. K. Batchelor, An Introduction to Fluid Dynamics, Cambridge University Press, Cambridge, UK, 1967.

[30] M. E. Erdoğan and C. E. İmrak, "On the properties of a special function defined by an integral," Mathematical and Computational Applications, vol. 11, no. 1, pp. 11-18, 2006.

[31] M. E. Erdoğan and C. E. İmrak, "Effects of the side walls on unsteady flow of a second grade fluid over a plane wall," International Journal of Non-Linear Mechanics, vol. 43, no. 8, pp. 779-782, 2008.

[32] C. J. Tranter, Integral Transforms in Mathematical Physics, John Wiley \& Sons, New York, NY, USA, 1956.

[33] R. M. Robinson, "The effects of a corner on a propagating internal gravity wave," The Journal of Fluid Mechanics, vol. 42, pp. 257-267, 1970.

[34] M. Abromowitz and I. A. Stegun, Eds., Handbook of Mathematical Functions, Dover, New York, NY, USA, 1965.

[35] H. Schlichting, Boundary Layer Theory, McGraw-Hill, New York, NY, USA, 1968.

[36] I. S. Grandshteyn and I. M. Ryzhik, Tables of Integrals, Series and Products, Academic Press, New York, NY, USA, 1965. 


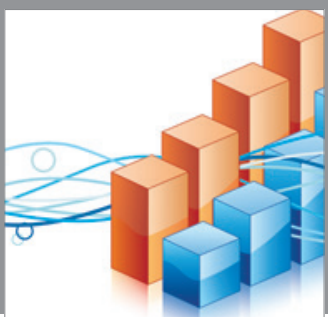

Advances in

Operations Research

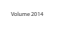

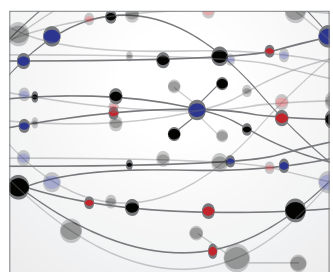

\section{The Scientific} World Journal
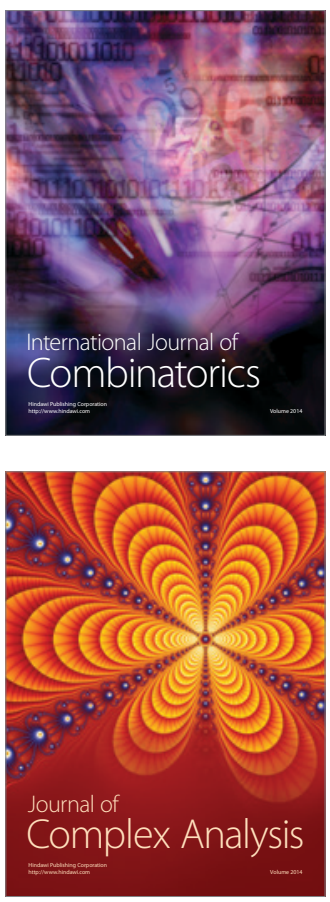

International Journal of

Mathematics and

Mathematical

Sciences
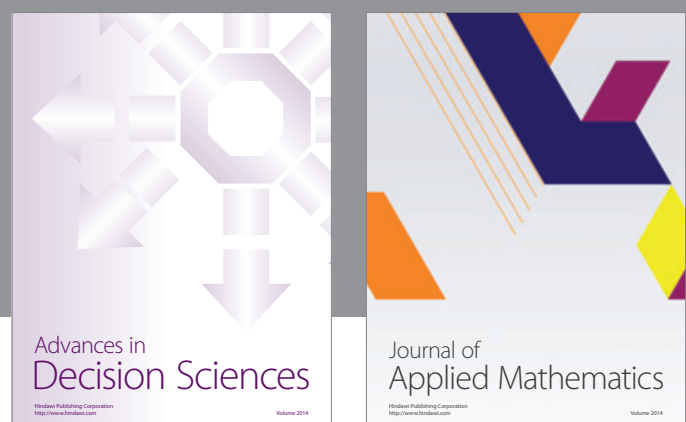

Journal of

Applied Mathematics
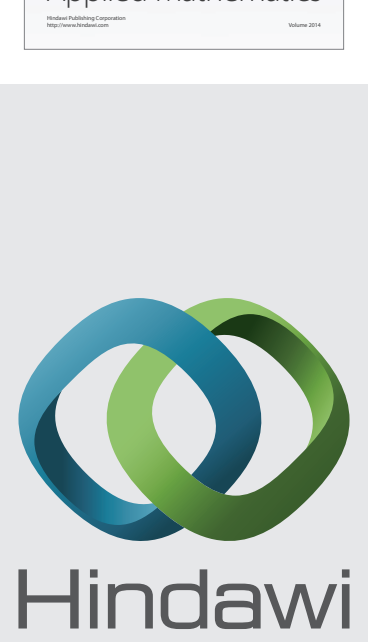

Submit your manuscripts at http://www.hindawi.com
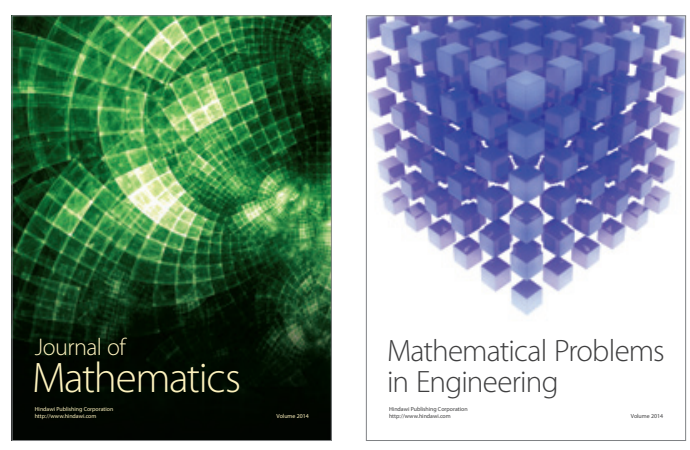

Mathematical Problems in Engineering
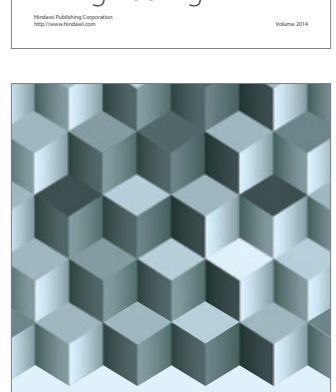

Journal of

Function Spaces
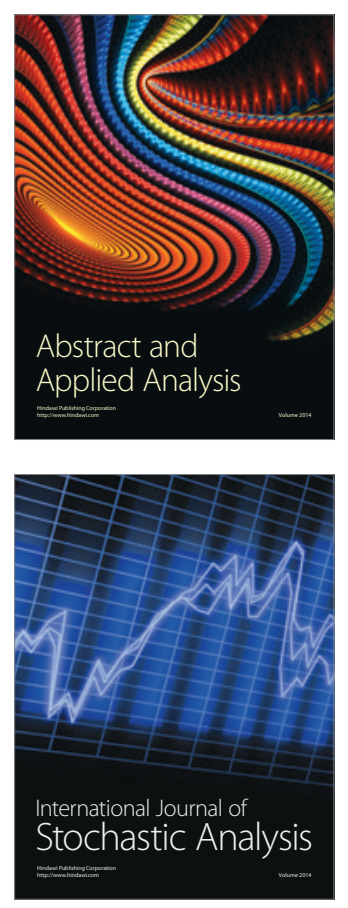

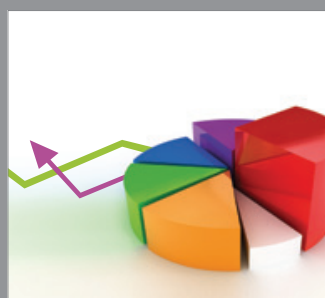

ournal of

Probability and Statistics

Promensencen
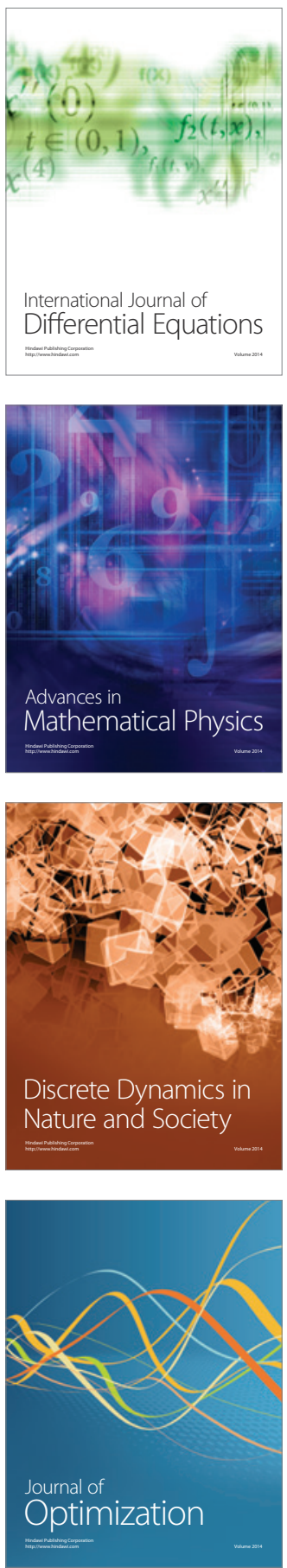